\title{
Robot Face Memorability is Affected by Uncanny Appearance
}

\author{
Allie R. Geiger \& Benjamin Balas
}

Author Note:

Benjamin Balas, Department of Psychology, Center for Visual and Cognitive Neuroscience, North Dakota State University.

Correspondence regarding this article should be addressed to Benjamin Balas, Department of

Psychology, North Dakota State University, Fargo, ND 58102

Contact: Benjamin.balas@ndsu.edu 


\section{Abstract}

Human face recognition is influenced by various social and environmental constructs. For example, both age and race can affect the likelihood that a human face will be correctly recalled. Interestingly, general face appearance (i.e. friendly or untrustworthy faces) can also influence memorability. As human-robot interaction (HRI) becomes more commonplace, understanding what factors influence face recognition for non-human social agents is increasingly important. In particular, while there is a growing literature comparing the recognition of real human faces to computer-generated face images, comparisons between human face processing and robot face processing are largely unexplored. Here, we examined how the uncanny/eeriness of robot-faces affects memorability by using a $2 \mathrm{AFC}$ old/new task with various robot faces. Participants rated robot and human faces on perceived uncanniness during a study phase and were subsequently given a surprise memory task with only a fraction of the previously-encountered robot faces. Our results suggest that robots who are rated as more uncanny are more memorable than those that do not elicit the eerie feelings that correspond with uncanny faces: The more uncanny the robot face, the more accurately and quickly they were recalled. We discuss these results in the context of the design of social agents for HRI and also vis-a-vis theories of human face recognition and memory. 


\section{Introduction}

Human-robot interactions (HRI) are becoming more prevalent in society. For example, social robots are being integrated into the medical field to provide therapeutic services to individuals on the autism spectrum (Diehl et al., 2012) and into elder living facilities to act as companions to elders (Broekens et al., 2009). Additionally, there have been more than 200 instances of robots being adopted into society, in places such as medical and educational facilities, as a direct result of the COVID-19 pandemic (Murphy et al., 2020). With the functionality of social robots rapidly increasing, investigating HRI in relation to human cognitive and sensory processing is vital to enhancing the effectiveness of robots in society.

One key component of successful social interaction is face recognition. In HRI, this means both that artificial social agents should be capable of remembering and recognizing the faces of humans that they interact with and that human abilities to recognize robot faces may affect the quality of human-robot social exchange. Methods to increase robots' ability to recognize human faces following brief interactions (Cruz et al., 2008) continue to improve, but our understanding of how humans process robot faces is comparatively less well-understood.The mechanisms underlying the memorability and recognition of robot faces may be different than those that support the processing of human faces and other artificial face stimuli. Even within the domain of human face stimuli, visual experience that favors specific categories defined by race (Zhao et al., 2014), and age (Rhodes \& Anastasi, 2012) ultimately limits visual capabilities for recognizing faces that have not been encountered as frequently. The most well-studied example of this phenomenon the other-race effect (ORE), which refers to a range of related outcomes favoring same-race faces over different-race faces, including recall accuracy (Meissner \& Brigham, 2001) and identity matching (Megreya, White \& Burton, 2011). Visual experience also affects face recognition abilities within majority categories: Limited exposure to faces during development as a result of living in a small community leads to poorer face learning and less distinct neural responses to faces and non-face objects (Balas \& Saville, 2015). Given that we expect most observers have far greater experience with human faces than robot faces, these results suggest that both behavioral and neural 
markers of face recognition abilities should reflect less effective and/or less efficient processing of robot faces.

Previous research indeed suggests that artificial faces, including other categories of synthetic faces like computer-generated images, dolls, and robots, are generally not recognized or remembered as well as human faces. For example, Balas and Pacella (2015), demonstrated that the memorability of computer-generated (CG) faces was worse than that of human faces, , even when the CG faces were created using specific human models. (Balas \& Pacella, 2015). In other words, participants saw the same people, though some were rendered into CG faces. The estimation of personality variables from faces, including perceived trustworthiness, is also disrupted when artificial face appearance is imposed by rendering computer-generated faces (Balas \& Pacella, 2017; Balas \& Tupa, 2018). Finally, Geiger \& Balas (2020) demonstrated that neural markers of face processing are less sensitive to artificial faces, with computer-generated faces and dolls generated object-like responses relative to human faces, but with robot faces yielding an intermediate response that suggests partial tuning of the human visual system for robot face appearance. Despite these differences in face processing, which include a categorical boundary between real and artificial faces (Looser \& Wheatley, 2010), some aspects of face processing appear to generalize across real and artificial face stimuli. Neural adaptation to face appearance, for example, is transferable between cartoon and real human faces (Chen et al., 2010), suggesting some shared mechanisms for these categories.

As a minority category in visual experience, robot faces are thus an interesting category of faces to explore in terms of visual recognition, including memorability, social judgments, and face matching and discrimination. A particularly interesting issue that must be taken into account when studying robot faces is the so-called Uncanny Valley, which refers to the potential for some robot faces to be perceived as unsettling, "creepy," or grotesque. Briefly, the Uncanny Valley refers to an observed pattern between the realism of a robot face and the extent to which that face is perceived as pleasant to look at and likable: As the humanness of robots increases, their likability tends to decrease, but when robot faces become extremely human-like, likability tends to increase (Mathur \& Reichling, 2015; Mori et al., 2012). In other 
words, the more human-like robot faces become, the less affable they appear to observers (Mathur \& Reichling, 2015) until some threshold of humanness is crossed, leading to increased likability. That nonlinear relationship, specifically the "dip" in likability between very unrealistic and very realistic humanoid robots (Figure 1), is known as the Uncanny Valley (UV). Looking at robot faces in the UV, observers may get eerie and unsettling feelings, which were initially hypothesized to be related to category confusion between human and robot labels, but which have most recently been demonstrated to be more closely related to varying realism across the robot's facial features (Chattopadhya \& MacDorman, 2011; Macdorman \& Chattopadhya, 2016). Besides leading to feelings of uneasiness, people tend to have negative attitudes towards uncanny robots (Kim et al., 2019) and in some cases, do not trust robots in the UV (Mathur \& Reichling, 2015). In the present study, we ask a simple question about robot face uncanniness and face recognition: Does the uncanniness of robots in the UV affect how they are remembered?

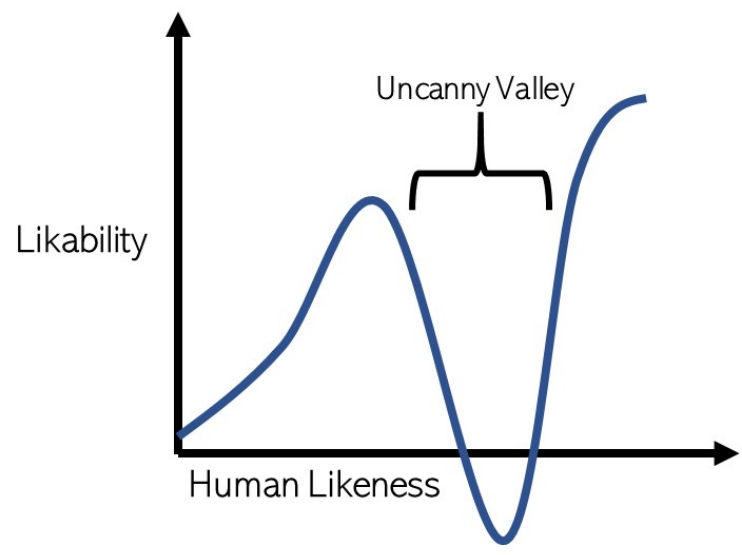

Figure 1. A schematic view of the relationship between Likability and Human Likeness. The Uncanny Valley refers to a theoretical dip in Likability as Human Likeness approaches veridical human appearance. Uncanniness may affect multiple aspects of face recognition, including memory for recently seen faces. 
Memorability is an intrinsic property of face images ( Bainbridge, Isola \& Oliva, 2013). That is, there is consistent variation in memorability across face images (some faces are remembered better than others) and robust test-retest reliability for the memorability of individual faces (Bainbridge et al., 2012). Moreover, the variability in face memory across a large set of images is not completely predicted by personal or social traits (Bainbridge, Isola \& Oliva, 2013), though there are clear relationships between typicality and memorability (Vokey \& Read, 1992) and also between trustworthiness and likability (Bainbridge, Isola \& Oliva, 2013; Rule et al., 2012). This means that while these factors influence memorability to some extent (typicality negatively, trustworthiness positively) memorability may also be a unique property that is not easily reduced to component traits like these. Regardless, face memorability can be learned from large collections of face images (Bylinskii et al., 2021), supporting both analyses of how various transformations of appearance may affect memorability (Bainbridge, 2016) and specific image manipulations that increase face memorability (Khosla et al., 2013). How should uncanny robot appearance affect memorability based on previous results? To our knowledge, the relationship between uncanniness and typicality has not be closely examined, but naively it seems like an uncanny face should be perceived as atypical (most faces are not creepy), but unlikable. These factors make opposite predictions regarding the memorability of face images, which makes it challenging to make a clear prediction regarding uncanny robot faces. Moreover, to the extent that memorability is a unique and intrinsic property of face images, variance beyond these factors likely contribute to the overall memorability of robot faces. It is therefore an open question whether robots as a stimulus category are subject to the effects seen in human and other artificial face memorability,, as what we know about human and artificial face memorability may not be generalizable to robots.

In the present study we conducted a simple behavioral experiment to investigate how memorability is affected by the 'uncanniness' of robots. Specifically, we hypothesized that a participant's accuracy to remember previously seen robot faces would be affected by face uncanniness, but were agnostic about the direction of the effect. We implemented this experiment using a large database of robot face images with accompanying metadata (Mathur et al., 2015) allowing us to select robot faces with a 
range of perceived human-like ratings and examine memorability as a function of uncanny appearance. Besides examining human performance with these images, we also evaluated how a convolutional neural net model of face memorability predicted the memorability of our stimulus set (Needell \& Bainbridge, 2021).

\section{Methods}

\subsection{Participants}

We recruited a sample of 69 (42 female) participants between the ages of 18-32 years old from the NDSU's Undergraduate Psychology Study Pool (administered by the SONA system) Upon signing up to participate, individual participants were asked to read a consent form and indicate their agreement to participate by continuing on to the main portion of the experiment.

\subsection{Stimuli}

We selected human and robot face images from the stimulus set used in Mathur et al. (2020). This stimulus set represents a validated face corpus of robot, humanoid robot, and human face images that vary in mechano-humanness (MH). To control for low-level differences between human and robot face images that might impact face memorability, we carried out a series of image processing steps designed to minimize such differences across all of our images. First, we resized each image to 300x300 pixels and converted them to grayscale using MATLAB's rgb2gray.m function. Next, we matched the power spectra and intensity histograms of all images using functions in the MATLAB SHINE Toolbox (Willenbockel et al., 2011). Our final stimulus set was composed of 204 stimuli, representing a range of $\mathrm{MH}$ values and with low-level properties matched across the entire stimulus set (Figure 2). 

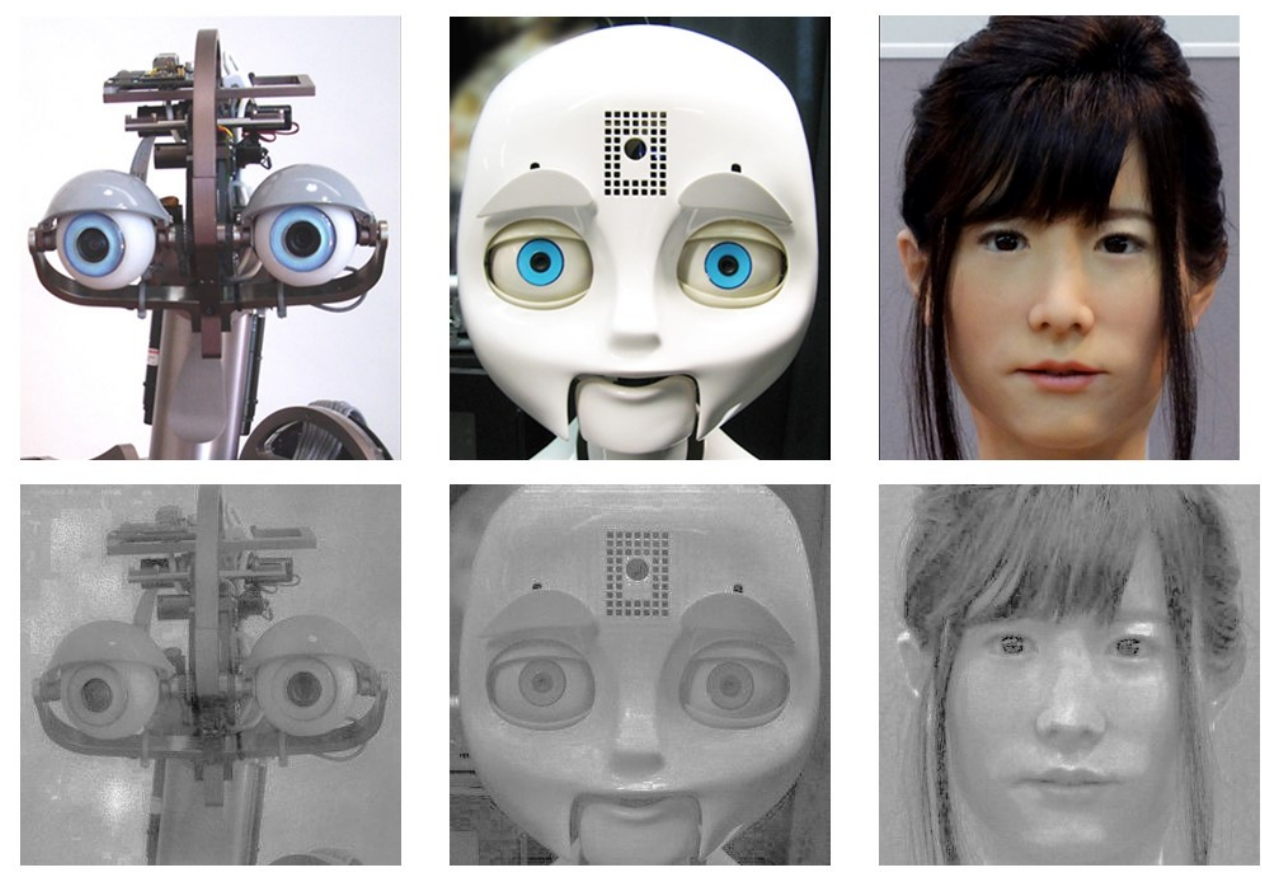

Figure 2. Example stimuli selected from Mathur et al. (2020) with mechano-humanness increasing from left-to-right. The top row depicts unprocessed stimuli, whereas the bottom row of stimuli have undergone desaturation and both intensity histogram matching and power spectrum normalization.

\subsection{Procedure}

All stimulus display and response collection routines were carried out using custom software written in PsychoPy (Peirce et al., 2019). Due to the COVID-19 pandemic, all testing was carried out online using the Pavlovia platform (https://pavlovia.org) beginning in January of 2021 and terminating in April of 2021. As such, we had limited control over the displays participants used to complete the study, which means image size, viewing distance, and other aspects of the testing scenario were variable across participants.

After providing demographic information about themselves including age, ethnicity, and gender, participants completed two different phases of our experiment: A study phase and a surprise memory test. 
During the study phase, participants rated 176 robot and human faces on a scale from zero to onehundred with endpoints labeled as "Not Uncanny or Creepy at all" and "Extremely Uncanny and Creepy." To ensure that participants understood the nature of uncanniness, the study phase was preceded by a short description of the Uncanny Valley (UV) along with example animated facial images that typically elicit the eerie feeling of the UV, and those that do not. We did not use robot face images in this example to avoid directly biasing participants' judgments during the study phase. We note that the Mathur et al. (2020) images include MH and likability metadata that accompany the stimuli, but collecting our own uncanniness ratings served two purposes: First, asking participants to provide uncanniness ratings ensures that participants attended to each image closely enough to determine their impressions of it. Second, these uncanniness ratings can be compared to the metadata provided by Mathur et al. (2020) to examine the relationship between their mechano-humanness and likability measures and an explicit judgment of uncanniness/creepiness. Participants were permitted to view each image for as much time as they liked, after which we asked them to provide their rating of uncanniness. Study images were presented in a pseudo-randomized order that differed for each participant. Further, participants were randomly assigned to one of two groups, which differed in terms of a subset of images presented during the study phase. While all participants were shown a common set of 148 distractor stimuli, a subset of 28 images were selected as the targets for our subsequent memory test. Participants in these two groups were presented with different sets of 28 target stimuli, each of which was matched to an image in the other set for mechano-humanness. This manipulation allowed us to minimize the contribution of any individual images in the target subset that may have either uniquely high or low memorability for idiosyncratic reasons.

Following the study phase, participants were asked to complete a surprise memory test during the second phase of the experiment. During this test phase participants were shown 28 side-by-side pairs of unedited robot faces (presented in full-color and without histogram/power-spectrum matching), one of which they 
had been shown in the study phase of the task and one which had not. Members of each pair were matched for mechano-humanness and the target/distractor assignment for each image pair was balanced across our two groups of participants. That is, the target images for one group were the distractor images for the other. Participants were instructed to use the right and left arrow keys to indicate which robot they had seen before as quickly as possible while still being accurate. The left-right presentation of each stimuli was randomized by functions in PsychoPy (Peirce et al., 2019). The full set of PsychoPy functions and stimuli can be found in a public repository maintained by the Open Science Framework: (https://osf.io/6u7g8/).

\section{Results}

\subsection{Pre-registered Analyses}

We divided our test phase stimulus set into two groups using a median split on z-scored 'uncanniness' ratings obtained during the study phase of the experiment, where one half of the stimuli were on average considered more uncanny than the other half. Specifically, we calculated within-participant z-scores for the uncanniness ratings obtained during the study phase and then averaged these per stimulus across all participants. The resulting average $\mathrm{z}$-scores were then used to determine a median value that we used to divide the stimulus set into a "more uncanny" and "less uncanny" group. Any missing values per participant were not included in either the z-score calculation or between-participant averaging. Participants were also excluded if they had abnormally fast response times $(0.3 \mathrm{~ms})$ or if they had a significant portion of missing data. Using JASP (JASP Team, 2020) we conducted t-tests comparing both accuracy and RTs between the groups above and below the median z-scores. This analysis revealed no significant difference in the two groups for accuracy $(\mathrm{t}(27)=0.208, \mathrm{p}=0.837)$ or $\mathrm{RTs}(\mathrm{t}(27)=1.433$, $\mathrm{p}=0.163$.) 


\subsection{Exploratory Analyses}

To further explore our data, we also chose to carry out a series of correlational analyses examining the relationships between recall accuracy, uncanniness, and related measures of stimulus appearance. Compared to our simple median-split analysis described above, these analyses consider recall and perceived stimulus properties continuously, giving us more power to see relationships between these variables.

\subsubsection{Perceived Uncanniness vs. Mechano-Humanness/Likability}

Mathur \& Reichling (2015) asked participants in their study to rate robot faces according to mechanohuman likeness (How much does this robot resemble person?) and likability (How much do you like this face?). Given that we directly asked participants to rate the uncanniness of each face, we examined the relationship between these two descriptors of face appearance and an explicit uncanniness judgment (Figure 3). This analysis revealed a clear U-shaped relationship between mechano-humanness and uncanniness, which is consistent with the classic description of the Uncanny Valley: Faces of intermediate mechano-human likeness are perceived as the most uncanny. Uncanniness and likability also exhibited a clear linear relationship (Spearman's rho $=-0.66, \mathrm{p}<0.001, \mathrm{n}=56$ ), such that more likable faces were perceived as less uncanny. 
Avg. Mechano-Human

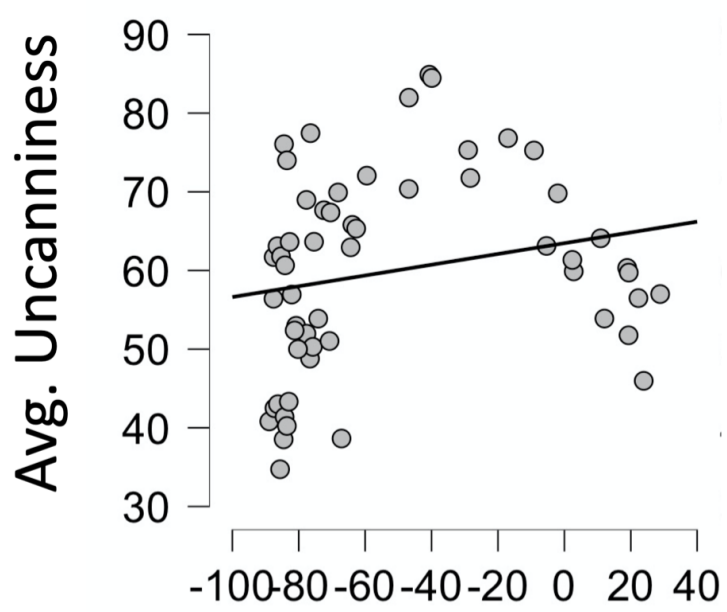

Avg. Likability

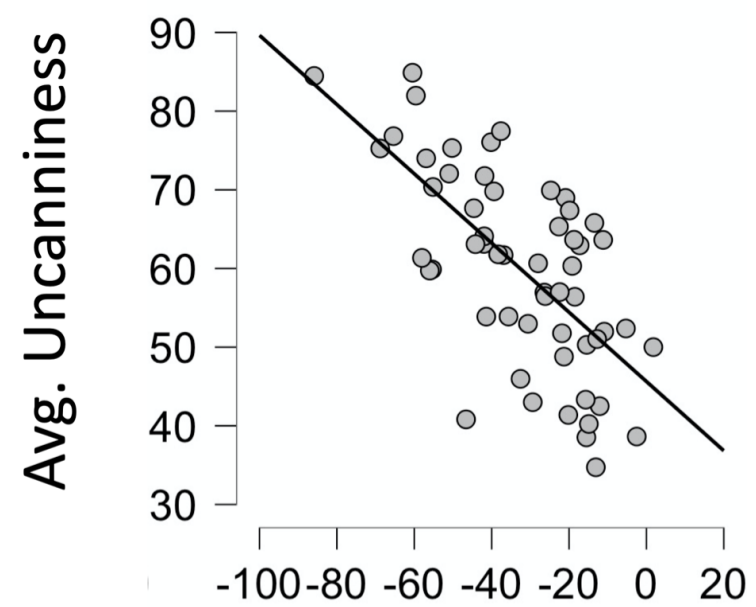

Figure 3 - Correlations between average uncanniness and average mechano-human likeness ratings (left panel) and likability (right panel). Perceived uncanniness is strongly related to both of these variables, but according to a quadratic and linear function respectively.

\subsubsection{Recall Accuracy vs. Perceived Uncanniness}

Next, we calculated the average uncanniness rating assigned to each test image during the study phase across all participants and also calculated the proportion of correct responses to each test image during the test phase across all participants. If perceived uncanniness does influence memorability, we should observe a significant correlation between these values. Using JASP (JASP Team, 2020), we observed that there was a significant correlation between the average ratings per stimulus and accuracy (Spearman's rho $=0.355, \mathrm{p}=0.007$ - see Figure 4). We also found that perceived uncanniness and RT were significantly correlated (Spearman's rho $=-0.455, \mathrm{p}<0.001$ ) such that more uncanny faces were responded to more quickly during the test phase. These results suggest that memorability is affected by uncanny face appearance, but that this is less evident in a group analysis than an item-based correlation analysis. 


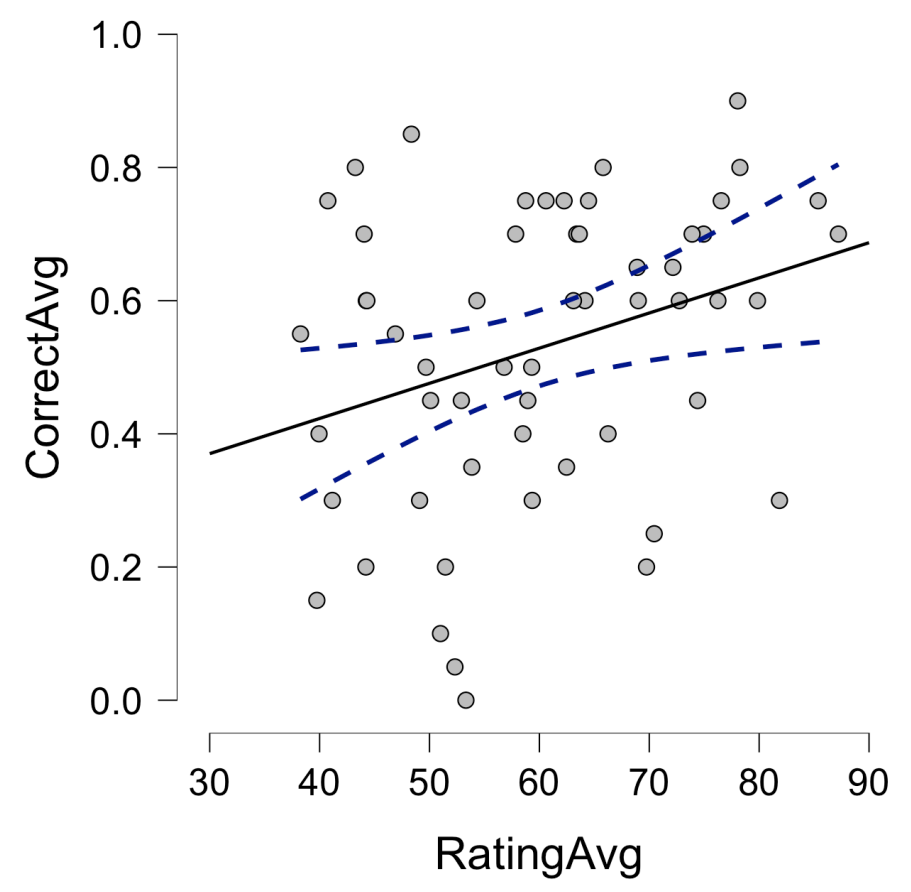

Figure 4. Average per stimulus memorability (y-axis - average proportion correct measured during test phase) as a function of perceived uncanniness (x-axis average "uncanniness" rating measured during study phase). We observed a significant correlation between these variables such that more uncanny faces were more accurately remembered.

\subsubsection{Recall accuracy vs. ResMem memorability}

Finally, we chose to examine how well recall accuracy in our task was predicted by image memorability as estimated using ResMem (Needell \& Bainbridge, 2021) a convolutional neural net model trained on a large database of natural images. We obtained estimated memorability scores per image using ResMem (https://brainbridgelab.uchicago.edu/resmem/) and compared these values to the observed recall accuracy in our task (Figure 5) . This correlation was not significant (Spearman's rho $=0.042, \mathrm{p}=0.759$ ), suggesting that ResMem's model of image memorability does not capture what makes these specific images memorable or not. 


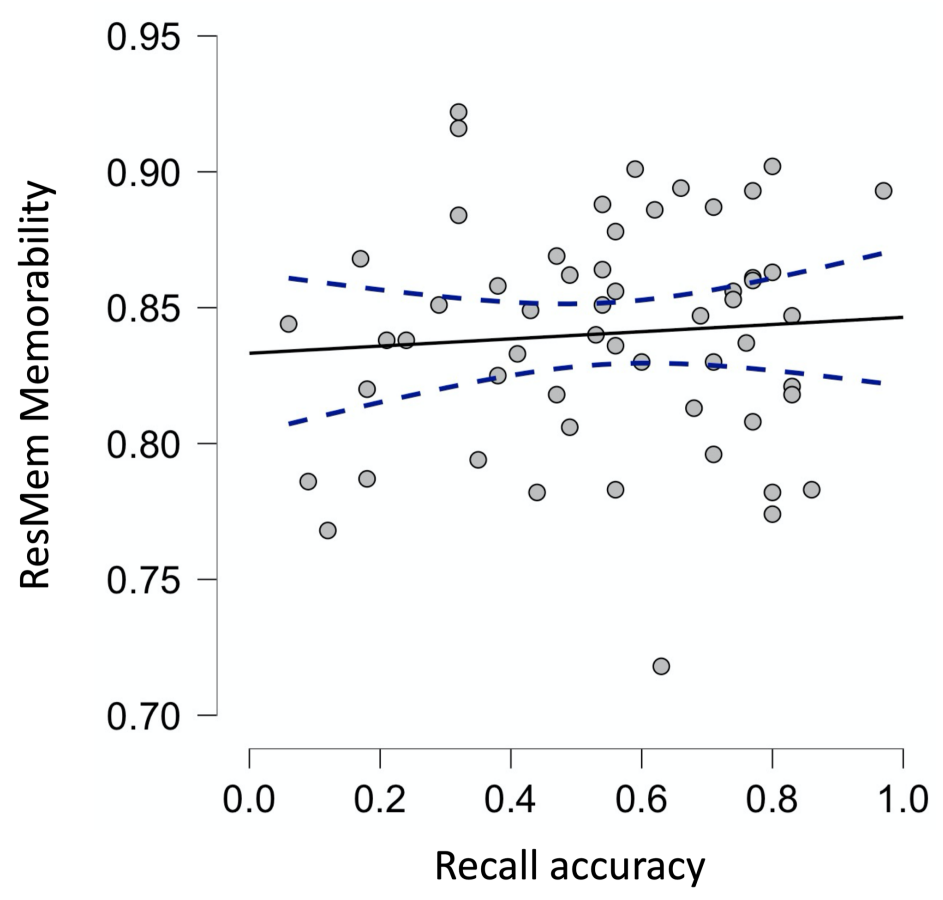

Figure 5 - Recall accuracy in our test phase plotted against estimated memorability obtained from ResMem. We find no relationship between these values, suggesting that ResMem does not capture variability related to uncanniness that contributes to robot face memory.

\section{Discussion}

In our experiment, our results reveal a relationship between perceived uncanniness and robot face memorability. Specifically, increased uncanniness was associated with increased memorability. While we did not observe this effect by considering the data in terms of discrete uncanny and non-uncanny groups, analyzing continuous variability in both uncanniness ratings and recall accuracy revealed a strong relationship between these variables. We also found that uncanniness negatively correlated with response time, suggesting that participants not only recalled uncanny faces more accurately, but they were faster to respond - a result that indicates high confidence in recall precision as uncanniness increases.. In terms of relationships between uncanniness and other variables related to memorability and social evaluation, we note that our uncanniness ratings were strongly correlated with likability. In turn, likability is closely correlated with trustworthiness (Rule et al., 2012), which is consistent with Mathur et al.'s (2015) results demonstrating that robot faces in the UV are perceived as less trustworthy. While we did not explicitly 
examine distinctiveness or typicality in this study (indeed, the question may be ill-posed for robot faces given that human faces dominate experience), this may be an indication that personal and social traits attributed to robot faces have a larger influence on cognitive and visual task performance than properties more closely related to image-level information.

This speculative conclusion is to some extent consistent with our analysis of the null relationship between ResMem's predictions of image memorability and recall accuracy in our task. Our results demonstrate that the underlying model of face memorability learned by ResMem does not generalize well to robot faces. By itself, this suggests that the image-level statistics that are applicable to human faces do not transfer directly to robot faces, which is perhaps not entirely unexpected given the large differences in robot face surface properties, face configuration, and other aspects of facial appearance. However, we also suggest that while ResMem's underlying model may implicitly reflect relationships between social variables like trustworthiness and image data (relationships which have been explicitly modeled and manipulated in human face perception experiments (Oosterhof \& Todorov, 2008)), the lack of a connection between robot appearance and perceived trustworthiness, likability, and other traits that predict memorability may be the cause of ResMem's inability to predict robot face recall here. Alternatively, without appealing to these social variables, it may also be the case that intrinsic memorability itself varies across robot faces in image space sufficiently differently than for human faces to make ResMem's estimates less applicable. Understanding the contribution of social traits and intrinsic memorability to robot face memory in the specific may be an important direction for future work, as both of these aspects of facial appearance may be computable by human observers but in fundamentally different ways than they are computed for human faces.

Overall, our results demonstrate that uncanny robot appearance does affect memorability and that it does so positively. This is in some ways a challenging result for designing robots that can engage in HRI effectively: The most memorable robots are the most unsettling. However, further examination of how intrinsic memorability varies across different robot faces may support domain-specific learning of 
how to increase the memorability of robot faces per Khosla et al. (2013) such that uncanny appearance is not an inevitable by-product. Also, by continuing to examine how variables like distinctiveness, likability, and memorability may interact with each other differently in the context of robot faces, shaping HRI based on human cognition and perceptual abilities and experience should lead to social agents that are more effective and easier to engage with.

\section{Acknowledgments}

We would like to thank Ilya Nudnou, Enrique Alvarez, Dan Gu and Ganesh Padmanabhan for their assistance with the implementation of our experiments and online recruiting and testing.

\section{References}

Bainbridge, W.A., Isola, P., Olivia, A. (2013). The Intrinsic Memorability of Face Photographs. Journal of Experimental Psychology, 142(4), 1323-1334.

Bainbridge, W.A., Isola, P., Blank, I., \& Oliva, A. (2012). Establishing a Database for Studying Human Face Photograph Memory. Cognitive Science, 34.

Bainbridge, W.A. (2017). The Memorability of People: Intrinsic Memorability Across Transformations of a Person's Face. Journal of Experimental Psychology: Learning, Memory, and Cognition, 43, 706-716.

Balas, B. \& Pacella, J. (2015). Artificial faces are harder to remember. Computers in Human Behavior, 52, 331-337.

Balas, B., \& Pacella, J. (2017). Trustworthiness perception is disrupted in artificial faces. Computers in Human Behavior, 77, 240-248. https://doi.org/10.1016/j.chb.2017.08.045 
Balas, B., \& Saville, A. (2017). Hometown size affects the processing of naturalistic face variability.

Vision research, 141, 228-236. https://doi.org/10.1016/j.visres.2016.12.005

Balas, B., Tupa, L., \& Pacella, J. (2018). Measuring social variables in real and artificial faces. Comput. Hum. Behav., 88, 236-243.

Broekens, J., Heerink, M., \& Rosendal, H. (2009). Assistive social robots in elderly care: A review. Gerontechnology, 8, 94-103.

Bylinskii, Z., Goetschalckx, L., Newman, A., \& Oliva, A. (2021). Memorability: An image-computable measure of information utility. ArXiv, abs/2104.00805.

Chattopadhyay, D. \& MacDorman, K.F. (2011). Familiar faces rendered strange: Why inconsistent realism drives characters into the uncanny valley. Journal of Vision, 16(11), $1-25$.

Chen, H., Russell, R., Nakayama, K., \& Livingstone, M. (2010). Crossing the 'uncanny valley': adaptation to cartoon faces can influence perception of human faces. Perception, 39(3), 378-386. https://doi.org/10.1068/p6492

Cruz, C., Sucar, L.E., \& Morales, E.F. (2008, September 17-19). Real-time face recognition for human-robot interaction. 2008 8th IEEE International Conference on Automatic Face \& Gesture Recognition, Amsterdam, Netherlands. DOI: 10.1109/AFGR.2008.4813386.

Diehl, J.J., Schmitt, L.M., Villano, M., \& Crowell, C.R. (2012). The clinical use of robots for individuals with autism spectrum disorders: A critical review. Research in Autism Spectrum Disorders, 6(1), 249-262.

Geiger, A.R. \& Balas, B. (2020). Not quite human, not quite machine: Electrophysiological responses to robot faces. bioRxiv, 1-36. DOI: 10.1101/2020.06.11.145979

Isola, P., Xiao, J., Torralba, A., \& Oliva, A. (2011). What makes an image memorable? CVPR, $145-152$.

JASP Team (2020). JASP (Version 0.14.1)[Computer software]. 
Khosla, A., Bainbridge, W.A., Torralba, A., \& Oliva, A. (2013). Modifying the Memorability of Face Photographs. 2013 IEEE International Conference on Computer Vision, 3200-3207.

Kim, S.Y., Schmitt, B.H., \& Thalmann, N.M (2019) Eliza in the uncanny valley:

Anthropomorphizing consumer robots increases their perceived warmth but decreases liking. Marketing Letters, 30, 1-12.

Looser, C. E., \& Wheatley, T. (2010). The tipping point of animacy. How, when, and where we perceive life in a face. Psychological science, 21(12), 1854-1862. https://doi.org/10.1177/0956797610388044.

MacDorman, K. F., \& Chattopadhyay, D. (2016). Reducing consistency in human realism increases the uncanny valley effect; increasing category uncertainty does not. Cognition, 146, 190-205.

Mathur, M.B., \& Reichling, D.B. (2015). Navigating a social world with robot partners: A quantitative cartography of the uncanny valley. Cognition, 146, 22-32.

Mathur, M., Reichling, D.B., Lunardinid, F., Geminianid, A., Antoniettid, A., Ruijtene, P.A.M., Levitanf, C.A., Naveg, G.,Manfredig, D., Bessette-Symonsh, B., Szutsi, A., \& Aczeli, B. (2020). Uncanny but not confusing: Multisite study of perceptual category confusion in the Uncanny Valley. Computers in Human Behavior, 103, 21-30.

Megreya, A. M., White, D., \& Burton, A. M. (2011). The other-race effect does not rely on memory: Evidence from a matching task. Quarterly journal of experimental psychology (2006), 64(8), 1473-1483. https://doi.org/10.1080/17470218.2011.575228

Meissner, C.A. \& Brigham, J.C. (2001). Thirty years of investigating the own-race bias in memory for faces. Psychology, Public Policy, \& Law, 7(1), 3-35.

DOI: $10.1037 / / 1076-8971.7 .1 .3$

Mori, M., MacDorman, K.F., \& Kageki, N. (2012). The uncanny valley [from the field]. IEEE Robotics \& Automation Magazine, 19(2), 98-100.

Murphy, R.R., Gandudi, V.B.M., \& Adams, J. (2020). Applications of robots for COVID-19 response. arXiv:2008.06976. 
Needell, C. D., \& Bainbridge, W. A. (2021). Embracing New Techniques in Deep Learning for Estimating Image Memorability. ArXiv:2105.10598 [Cs]. http://arxiv.org/abs/2105.10598.

Oosterhof, N. N., \& Todorov, A. (2008). The functional basis of face evaluation. Proceedings of the National Academy of Sciences of the United States of America, 105(32), 11087-11092. https://doi.org/10.1073/pnas.0805664105

Peirce, J. W., Gray, J. R., Simpson, S., MacAskill, M. R., Höchenberger, R., Sogo, H., Kastman, E., \& Lindeløv, J. (2019). PsychoPy2: experiments in behavior made easy. Behavior Research Methods. 10.3758/s13428-018-01193-y

Rule, N.O., Slepian, M.L., \& Ambady, N. (2012). A memory advantage for untrustworthy faces. Cognition, 125(2), 207-218.

Rhodes, M. G., \& Anastasi, J. S. (2012). The own-age bias in face recognition: A meta-analytic and theoretical review. Psychological Bulletin, 138(1), 146-174. https://doi.org/10.1037/a0025750.

Seyama, J., \& Nagayama, R.S. (2007). The Uncanny Valley: Effect of Realism on the Impression of Artificial Human Faces. PRESENCE: Teleoperators and Virtual Environments, 16, 337-351.

Vokey, J.R., \& Read, J. (1992). Familiarity, memorability, and the effect of typicality on the recognition of faces. Memory \& Cognition, 20, 291-302.

Zhao, M., Hayward, W. G., \& Bülthoff, I. (2014). Holistic processing, contact, and the other-race effect in face recognition. Vision research, 105, 61-69. https://doi.org/10.1016/j.visres.2014.09.006 